\title{
Outcomes of endoscopic submucosal dissection (ESD) during live endoscopy events (LEE) - a 13-year follow-up
}

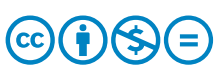

\author{
Authors \\ Joerg Marienhagen², Helmut Messmann' \\ Institutions \\ 1 Department of Gastroenterology, Universitätsklinikum \\ Augsburg, Germany \\ 2 Faculty of Medicine, University of Augsburg, Augsburg, \\ Germany
}

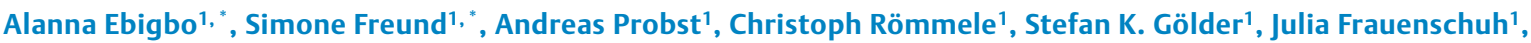

submitted 24.4.2019

accepted after revision $\quad 30.9 .2019$

\section{Bibliography}

DOI https://doi.org/10.1055/a-1035-9240 |

Endoscopy International Open 2019; 07: E1723-E1728

(c) Georg Thieme Verlag KG Stuttgart · New York eISSN 2196-9736

Corresponding author

Dr. Alanna Ebigbo, Universitätsklinikum Augsburg,

Stenglinstr. 2, 86156 Augsburg, Germany

Fax: +0049-821-400-2748

Alanna.ebigbo@uk-augsburg.de

\section{ABSTRACT}

Background and study aims There are no data showing the outcome of ESD during live endoscopy events (LEE). ESD performed during the Augsburg Endo-Update LEE were compared with matched routine procedures with the aim of demonstrating non-inferiority of LEE ESD.

Patients and methods ESD performed during the EndoUpdate between 2006 and 2018 were reviewed. The controls were routine procedures matched according to age, location and lesion size. Resection, recurrence, survival and complication rates, procedure time and propofol sedation were assessed. Clinically relevant margins were assumed for resection and complication rates, procedure time and propofol sedation quantity.

Results Thirty-eight ESD were performed in the given time period, and were compared with 38 matched routine ESD. En bloc and curative resection rates in the LEE group and in the control group were $100 \%$ and $87 \%$ as well as $84 \%$ and $71 \%$ respectively, while procedure times were 135 and 125 minutes, respectively. Non-inferiority was demonstrated for resection rates and procedure time. The complication rate was lower in the LEE group as compared with the control group ( $5 \%$ vs $13 \%$ ) while propofol sedation was similar in both groups ( $863 \mathrm{mg}$ vs $872 \mathrm{mg}$ ). Recurrence and 5-year survival rates for both groups were $4 \%$ vs $0 \%$ and $70 \%$ vs $65 \%$ respectively.

Conclusions The resection rate and procedure time of ESD during LEE was non-inferior to those of routine ESD procedures. Comparison of the complication rates, however, was inconclusive owing to the low patient number and complication risk in both groups.

\section{Introduction}

The number of live endoscopy events (LEE) has increased considerably over the last 20 years, and in most cases, they provide an excellent opportunity for physicians and nurses to witness state-of-the-art endoscopic procedures as well as new and innovative developments. However, a number of ethical and patient-related issues have been raised, especially concerning complications, patient outcome as well as success rate. Various factors could have a negative influence on the outcome of pro-

\footnotetext{
* These authors contributed equally.
}

cedures during LEE, including the fact that the operator has to concentrate on teaching and treatment simultaneously. Also, communication difficulties between the operator and the host staff as well as the stress of performing in front of a live audience could influence performance of the examiner [1]. Publications on endoscopic retrograde cholangiopancreatography (ERCP) during LEE have shown similar complication and success rates compared to routine procedures [1-3].

These issues prompted the European Society of Gastrointestinal Endoscopy (ESGE) to release a position statement on live endoscopy events in which it is stated that the primary utility of LEE is the educational value for the audience, and patients 
- Table 1 Matched parameters for both study groups.

\begin{tabular}{|c|c|c|}
\hline & LEE $(n=38)$ & Control $(n=38)$ \\
\hline Age (years) & Mean: 67 (range 24-84) & Mean: 65 (range 28-89) \\
\hline Sex (female/male) & $9 / 29$ & $13 / 25$ \\
\hline \multirow[t]{5}{*}{ Location } & Esophagus/esophagogastric junction $(n=11,29 \%)$ & Esophagus/esophagogastric junction $(n=11,29 \%)$ \\
\hline & Stomach $(n=10,26 \%)$ & Stomach $(n=10,26 \%)$ \\
\hline & Kolon $(n=4,11 \%)$ & Kolon $(n=4,11 \%)$ \\
\hline & Sigma $(n=2,5 \%)$ & Sigma $(n=2,5 \%)$ \\
\hline & Rectum $(n=11,29 \%)$ & Rectum ( $n=11,29 \%)$ \\
\hline \multirow[t]{3}{*}{ Size } & $1-2 \mathrm{~cm}(\mathrm{n}=1,3 \%)$ & $1-2 \mathrm{~cm}(\mathrm{n}=1,3 \%)$ \\
\hline & $2-4 \mathrm{~cm}(n=14,37 \%)$ & $2-4 \mathrm{~cm}(n=14,37 \%)$ \\
\hline & $>4 \mathrm{~cm}(\mathrm{n}=23,60 \%)$ & $>4 \mathrm{~cm}(\mathrm{n}=23,60 \%)$ \\
\hline
\end{tabular}

should not expect an additional benefit of being treated during a LEE compared to a routine setting [4].

An important aspect of LEE is that challenging procedures are often chosen for demonstration. The reason for this may be to educate physicians witnessing the LEE or also that experts especially proficient in these procedures are available during the LEE. One such procedure is endoscopic submucosal dissection (ESD) of early gastrointestinal lesions. ESD, developed in Japan, is an extremely tedious and difficult to learn endoscopic procedure with a long learning curve. There are no data or evidence showing the outcome of ESD during LEE.

In this paper, we present a retrospective match-controlled study comparing the outcome of ESD patients of the Universitätsklinikum Augsburg (UKA) treated during the live endoscopy event, Endo-Update, from 2006 to 2018. The primary aim of the study was to determine the success rate and safety of ESD during LEE.

Endo-Update is held annually and was initiated in 2006 with the primary aim of demonstrating novel and innovative endoscopic interventions in a real-life setting. Endoscopic cases at the Endo-Update are treated by world-class experts, and lesions are chosen especially for educational purposes. Procedures are shown via satellite to about 1000 observing physician-gastroenterologists. Because of the availability of experts during Endo-Update, cases selected for demonstration often tend to be more challenging and demanding. However, emphasis is placed on demonstrating technical and educational aspects of each procedure.

The UKA is a high-volume ESD center with an annual case load of about 200 ESD procedures performed by three endoscopists. The first ESD procedure in the UKA was conducted in 2003.

\section{Patients and methods}

All patients treated with ESD $(n=38)$ during Endo-Update were included in the study. The control group $(n=38)$ were patients treated during the same period in the endoscopy unit of the UKA by local endoscopists. Similar to the methodology adopted by Schmit et al, ESD cases performed after each Endo-Update were evaluated chronologically until the first best match was identified [1]. This patient was then included in the control group. This procedure was conducted for all Endo-Update patients (LEE group) during the period from 2006 to 2018. The control group was matched with the LEE group according to age of patients, size and location of lesions ( $\bullet$ Table 1$)$. Lesion size was broken into three categories: 0 to $2 \mathrm{~cm}, 2$ to $4 \mathrm{~cm}$ and larger than $4 \mathrm{~cm}$. Lesions larger than $4 \mathrm{~cm}$ were matched with lesions that were $\pm 1 \mathrm{~cm}$ larger or smaller. No recurrent lesions were included in the LEE group, which meant that this was not a matching criterion. All patients had an American Society of Anesthesiology (ASA) score of I or II.

Rates of en-bloc and curative resection, survival, recurrence, and complications, and examination time and quantity of propofol sedation were evaluated and compared between both groups using a clinically relevant, non-inferiority margin.

En-bloc resection was defined as complete resection of the tumor or lesion in one piece.

Curative resection was defined as tumor-free lateral and vertical margins, without lymphatic or vascular involvement and a maximum submucosal invasion depth of $\leq 1000 \mu \mathrm{m}$ for the colorectum and $\leq 500 \mu \mathrm{m}$ for gastric and esophageal adenocarcinoma while squamous cell carcinoma of the esophagus was limited to the upper $(\mathrm{m} 1)$ or middle $(\mathrm{m} 2)$ mucosal layer $[5,6]$.

Complications and adverse events (AE) were defined according to a lexicon of AE described by Cotton et al [7]. These included not just complications directly linked to the ESD intervention, such as perforation, but also others associated with sedation or anesthesia. Postinterventional bleeding was defined as presence of hematemesis and/or melena or a drop in the he- 
- Table 2 Comparison of resection rates, procedure time, propofol sedation and complication rate between the LEE group and the matched-control group.

\begin{tabular}{|c|c|c|c|}
\hline & LEE; $n=38$ & Control; $\mathbf{n}=\mathbf{3 8}$ & Difference $(95 \%-\mathrm{Cl})$ \\
\hline En bloc resection & $100 \% ;(38 / 38)$ & $87 \% ;(33 / 38)$ & $13 \%(2.5,27.8)$ \\
\hline Curative resection & $84 \% ;(32 / 38)$ & $71 \% ;(27 / 38)$ & $13 \%(-6.1,32.1)$ \\
\hline \multicolumn{3}{|l|}{ Procedure time } & \multirow[t]{3}{*}{$10(-19.6,39.6)$} \\
\hline - Mean (min) & 135 & 125 & \\
\hline - Range (min) & $40-210$ & $50-375$ & \\
\hline \multicolumn{3}{|l|}{ Propofol sedation } & \multirow[t]{3}{*}{$9(-313.7,295.7)$} \\
\hline - Mean (mg) & 863 & 872 & \\
\hline - Range (mg) & $120-2000$ & $100-2150$ & \\
\hline Complication & $5 \% ;(2 / 38)$ & $13 \% ;(5 / 38)$ & \multirow[t]{4}{*}{$8 \%(-20.8,5.0)$} \\
\hline Bleeding & $2(5 \%)$ & $3(8 \%)$ & \\
\hline Perforation & $0(0 \%)$ & $1(3 \%)$ & \\
\hline NSTEMI & $0(0 \%)$ & $1(3 \%)$ & \\
\hline
\end{tabular}

moglobin level of $>2 \mathrm{~g} / \mathrm{dL}$ which led to re-endoscopy for bleeding control.

Examination time was defined as time from insertion of the endoscope to completion of resection.

The quantity of propofol was measured in milligrams. Propofol sedation was measured only for patients receiving deep intra-venous sedation.

A recurrence was defined as discovery of a new lesion during a surveillance endoscopy at the same location where the previous ESD was performed.

The overall survival rate was stated as a 5 -year survival rate, which was the percentage of the patients, who were alive 5 years after ESD treatment.

Confidence intervals for differences between the study groups were computed with NCSS Statistical Software 2019. A clinically relevant effect for the study parameters was defined as follows: a difference in examination time of at least 60 minutes, a difference in propofol sedation quantity of at least $200 \mathrm{mg}$, a difference in curative or en bloc resection rate of $5 \%$ and a difference in complication rate of $5 \%$. Non-inferiority was achieved when the confidence intervals excluded the clinically relevant effect. As such, non-inferiority margins were +60 (procedure time), +200 (propofol sedation), -5 (resection rate) and +5 (complication rate). Non-inferiority margins were chosen as above because it was assumed that for LEE, cases tended to be more challenging and also endoscopists had to explain their procedures to the audience during LEE which would be more time-consuming. It was assumed that a margin of 60 minutes, 1 ampoule of propofol $(200 \mathrm{mg})$ or a difference of $5 \%$ in resection and complication rate was acceptable.

The learning curve of local ESD endoscopists over the 13year study period was also taken into consideration by dividing the study period into two halves. The first half included proce- dures performed from 2006 to 2012 while the second half was from 2013 to 2018. For this subgroup analysis, computation of confidence intervals was not done due to the low patient number.

\section{Results}

Thirty-eight ESD procedures were performed between 2006 and 2018 during the Augsburg Endo-Update LEE. Thirty-eight ESD procedures done outside the LEE (control group) were matched using the parameters described above ( $\downarrow$ Table 1 ). All 38 ESDs in the control group were performed by local endoscopists. In the LEE group, $82 \%(n=31)$ of ESD procedures were done by Japanese ESD experts while $18 \%(n=7)$ were done by two local endoscopists. In the first half of the study period (2006 to 2012), 20 ESD procedures were performed while 18 ESD procedures were conducted in the second half (2013 to 2018).

En bloc resection was achieved in $100 \%(n=38)$ in the LEE group and in $87 \%(n=33)$ in the control group. The clinically relevant effect and the non-inferiority margin were below the lower limit of the confidence intervals ( $\triangleright$ Table 2 ).

The overall curative resection rate was $84 \%(n=32)$ in the LEE group and $71 \%(n=27)$ in the control group. The clinically relevant effect and the non-inferiority margin were at the lower limit of the confidence intervals ( $\triangleright$ Table 2 ).

Two patients (5\%) in the LEE group and five patients (13\%) in the control group experienced a complication ( $\vee$ Table 2 ). Two patients $(5 \%)$ in the LEE group and 3 patients $(8 \%)$ in the control group had postinterventional bleeding with need for reendoscopy for bleeding control. No patients $(0 \%)$ in the LEE group and one patient ( $3 \%$ ) in the control group experienced a perforation in the upper colon that led to surgery. One patient 
(3\%) in the control group developed a myocardial infarction (non-ST segment elevation MI [NSTEMI]) within 48 hours after the endoscopic procedure. No other complications were observed. The clinically relevant effect and the non-inferiority margin were within the confidence intervals ( $>$ Table 2 ).

Median procedure time between the LEE group (135 minutes) and the control group (125 minutes) was similar. The clinically relevant effect and the non-inferiority margin were excluded by the confidence intervals ( $\bullet$ Table 2 ).

Propofol sedation was used in $55 \%$ of interventions $(n=21)$ in the LEE group and $71 \%(n=27)$ in the control group. Mean values of 863 and $827 \mathrm{mg}$ of propofol were used in the LEE group and the control group, respectively. The quantity of propofol per minutes was $6.4 \mathrm{mg} / \mathrm{min}$ in the LEE group and $7.0 \mathrm{mg} /$ min in the control group. The clinically relevant effect and the non-inferiority margin were within the confidence intervals ( Table 2).

Surveillance endoscopies were performed after 6 and 18 months. Of the patients in the LEE group, $96 \%(n=26 / 27)$ were recurrence-free after 6 months and $95 \%(n=20 / 21)$ after 18 months ( $>$ Table 3 ). Unfortunately, $29 \%$ of the patients $(n=$ $11 / 38)$ were lost to follow-up after 6 months and $50 \%(n=17 \mid$ 34) after 18 months in the LEE group. Four patients are still awaiting the 18 -month surveillance.

In the control group, $100 \%$ of patients $(n=24 / 24)$ were recurrence-free after 6 months and $100 \%(n=19 / 19)$ after 18 months. Of the patients, $37 \%(n=14 / 38)$ were lost to followup after 6 months and $56 \%(n=19 / 34)$ after 18 months. Four patients are still awaiting the 18 -month surveillance. There was no documented recurrence in the control group.

Retrospective information as regards the 5-year survival rate ( $\triangleright$ Table 3) was available for 23 of 38 patients in each group. In the LEE group, the 5 -year survival rate was $70 \%(n=16 / 23)$ while in the control group the 5-year survival rate was $65 \%$ ( $n$ $=15 / 23$ ).

Of the patients in the LEE group, $13 \%(n=3 / 23)$ died within 5 years after the intervention. Cause of death was not related to ESD or to disease for which ESD was performed.

In the control group, three patients died within 5 years, two of them not related to the disease for which ESD was performed. One patient died of metastatic esophageal carcinoma after initially curative intended ESD followed by radiochemotherapy 24 months after ESD-intervention. Four patients in the LEE group and five patients in the control group were lost to follow-up.

Twenty procedures (53\%) were conducted from 2006 to 2012 and 18 procedures from 2013 to 2018 (47\%) in both groups ( $\triangleright$ Table 4$)$. Curative resection rates improved from the first study period to the second study period ( $60 \%$ vs $75 \%$ in the control group and $83 \%$ vs $94 \%$ in the LEE group).

The complication rate during the first study period (2006 to 2012) was lower in the LEE group than in the control group (5\% vs $15 \%$ ) with three cases of postinterventional bleeding in the control group (15\%) and one case of bleeding in the LEE group (5\%). In the second study period, the complication rate was $6 \%$ $(1 \times$ bleeding $)$ in the LEE group and $11 \%(1 \times$ perforation, $1 \times$ NSTEMI) in the control group.
- Table 3 Comparison of recurrence and survival rates between the study groups.

\begin{tabular}{|l|l|l|}
\hline Recurrence & LEE & Control \\
\hline After 6 months & $4 \%(1 / 27)$ & $0 \%(0 / 27)$ \\
\hline After 18 months & $6 \%(1 / 17)$ & $0 \%(0 / 17)$ \\
\hline 5-year survival rate & LEE $\mathbf{n = 2 3}$ & Control $\mathbf{n}=\mathbf{2 3}$ \\
\hline & $\mathbf{7 0 \% ( 1 6 / 2 3 )}$ & $65 \%(15 / 23)$ \\
\hline & & \\
\hline LEE, live endoscopy event & &
\end{tabular}

\section{Discussion}

This is the first report showing the outcome of ESD procedures during a live endoscopy event (LEE). Various publications have shown that ERCP can be done safely and successfully, however, there are no data on ESD during a LEE. ESD is a tedious and difficult-to-learn procedure that originated in Japan and gradually spread to Europe and other Western regions [8,9].

ESD requires a high level of proficiency and skill, and the question about whether such an advanced and challenging procedure can be safely demonstrated at a LEE is important, especially considering the growing number of LEE. In ESD, the complex interplay between the operator and assistance personnel is crucial for a successful outcome. During LEE, the understanding between a foreign operator and the home personnel may be hindered, for example, by language barriers.

In our retrospective match-control study, we have shown that ESD procedures carried out during Endo-Update were non-inferior to those performed on routine controls in terms of resection rates and procedure time. The definition of a clinically relevant effect in a retrospective study design may appear arbitrary or even biased towards non-inferiority. However, the margins chosen for this study were based on statistical reasoning as well as clinical judgement. Non-inferiority was also shown for procedure time between the Endo-Update and control groups. The explanation may be that although the experts performing the ESD during Endo-Update may have spent more time lecturing to the audience or even waiting to demonstrate interesting aspects of a given procedure, they were still able to make up for this "lost time" due to their high level of skill and proficiency. Recurrence and overall 5-year survival rates were similar in both groups, however, owing to the low patient number, confidence intervals were not computed for these parameters.

Verbal and non-verbal communication necessary for a successful ESD did not seem to have a relevant effect on the performance of the foreign operators. A possible explanation for this may be the high level of expertise and proficiency of the foreign operators as well as the training and education of the assistance personnel. Furthermore, foreign operators were able to influence the choice of lesions and had sufficient time to assess their cases before the Endo-Update. Pre-interventional preparation took place together with the home team and involved clear communication on choice of equipment, electro- 
- Table 4 Comparison of resection rates, procedure time, propofol sedation, and complication rates between study groups during the two study periods.

\begin{tabular}{|c|c|c|c|c|c|c|}
\hline & \multicolumn{3}{|c|}{$2006-2012(n=20)$} & \multicolumn{3}{|c|}{$2013-2018(n=18)$} \\
\hline & LEE & Control & Difference & LEE & Control & Difference \\
\hline En bloc resection & $100 \%(20 / 20)$ & $90 \%(18 / 20)$ & $10 \%$ & $100 \%(18 / 18)$ & $83 \%(15 / 18)$ & $7 \%$ \\
\hline Curative resection & $75 \%(15 / 20)$ & $60 \%(12 / 20)$ & $15 \%$ & $94 \%(17 / 18)$ & $83 \%(15 / 18)$ & $11 \%$ \\
\hline Complication & $5 \%(1 / 20)$ & $15 \%(3 / 20)$ & \multirow[t]{4}{*}{$10 \%$} & $6 \%(1 / 18)$ & $11 \%(2 / 18)$ & \multirow[t]{4}{*}{$5 \%$} \\
\hline Bleeding & $5 \%(1 / 20)$ & $15 \%(3 / 20)$ & & $6 \%(1 / 18)$ & $0 \%(0 / 18)$ & \\
\hline Perforation & $0 \%(0 / 20)$ & $0 \%(0 / 20)$ & & $0 \%(0 / 18)$ & $6 \%(1 / 18)$ & \\
\hline NSTEMI & $0 \%(0 / 20)$ & $0 \%(0 / 20)$ & & $0 \%(0 / 18)$ & $6 \%(1 / 18)$ & \\
\hline \multicolumn{7}{|l|}{ Procedure time } \\
\hline - Mean (min) & 129 & 134 & \multirow[t]{2}{*}{5} & 140 & 119 & \multirow[t]{2}{*}{21} \\
\hline - Range (min) & 75-195 & $80-225$ & & $40-210$ & $50-375$ & \\
\hline \multicolumn{7}{|l|}{ Propofol sedation } \\
\hline - Mean (mg) & 837 & 839 & \multirow[t]{2}{*}{2} & 892 & 913 & \multirow[t]{2}{*}{21} \\
\hline - Range (mg) & $350-2000$ & $120-2150$ & & $120-1920$ & $100-1300$ & \\
\hline
\end{tabular}

surgical settings, ESD resection strategy, and risk management plan in case of an AE such as bleeding or perforation. Finally, Japanese operators were often accompanied by a second physician who supervised the assistance personnel before, during and after the ESD procedures. These points may have influenced the smoothness of the ESD procedures and also had an educational benefit for the home team.

Over the course of time, local endoscopists improved their level of proficiency with improved resection and complication rates ( $\downarrow$ Table 4 ). Again, due to the low patient number in the subgroup, confidence intervals were not computed but at least the tendency can be appreciated. In a recent meta-analysis, it was shown that the level of proficiency in ESD remains higher in Asian countries as compared to the West [10]. However, it also seems to be that ESD endoscopists in the West have improved their skills considerably in the past decade. Could this improvement be attributed, also, to LEE? The European Society of Gastrointestinal Endoscopy (ESGE) position statement on live endoscopy events states that the primary utility of LEE is the educational value [4]. The educational benefits of LEE are difficult to quantify from the data shown here. At least, there may have been an additional educational benefit for the local experts and their endoscopy assistance because all procedures performed by Japanese experts during the Endo-Update were accompanied by the local staff of the hospital.

A major limitation of this study is the low patient number as well as the retrospective nature of the study. A higher number of patients would have narrowed the confidence intervals, thereby improving the statistical power of the study. This was obvious in the study parameters (propofol sedation and complication rate) in which the confidence intervals were not able to exclude the clinically relevant effects, thus making a compari- son of these study outcomes inconclusive. In a prospective approach, other aspects of LEE, especially time spent on waiting "to go on-air," could have been assessed. Also, a more accurate match-control using other parameters such as morphology and accessibility (SMSA classification) could have been achieved [11]. Also, it was not possible to evaluate the amount of additional sedatives, such as midazolam or opiates, given to patients receiving propofol sedation. The fact that the majority of the ESD procedures during the Endo-Update were performed by Japanese experts is a bias in itself, and may lead to a question about whether the same results would hold for local endoscopists performing ESD during a LEE. The excellent outcomes of the en bloc resection rates with a tendency towards statistical significance underscore the expertise of the endoscopists who performed the ESD procedures in the LEE group in this study. From this study, it is obvious that whoever is chosen to perform an ESD during a LEE must have a high level of proficiency, confidence and experience.

\section{Conclusion}

Based on the data from this study, we can rightfully conclude that ESD procedures performed during LEE are non-inferior to routine procedures in terms of resection rate and procedure time. This may have an impact on endoscopy society guidelines around LEEs, and is also an important point for informed consent of patients undergoing an ESD during a LEE. However, a higher number of patients needs to be assessed, if possible, in a multicenter approach, to guarantee sufficient statistical power to make a conclusion on other study outcomes including complication rates. 


\section{Acknowledgements}

The authors acknowledge G. Braun, C. Fleischmann, R. Hieber, G. Jechart, S. Nagl, T. Oyama, A. Schneider, E. Schnoy, J. v. Rüden, A. Takahashi, C. Vollmer. T. Weber, and N. Yahagi.

Competing interests

None

\section{References}

[1] Schmit A, Lazaraki G, Hittelet A et al. Complications of endoscopic retrograde cholangiopancreatography during live endoscopy workshop demonstrations. Endoscopy 2005; 37: 695-699

[2] Liao Z, Li ZS, Leung JW et al. How safe and successful are live demonstrations of therapeutic ERCP? A large multicenter study Am J Gastroenterol 2009; 104: 47-52

[3] Ridititid W, Rerknimitr R, Treeprasertsuk S et al. Outcome of endoscopic retrograde cholangiopancreatography during live endoscopy demonstrations. Surg Endosc 2012; 26: 1931-1938
[4] Dinis-Ribeiro M, Hassan C, Meining A et al. Live endoscopy events (LEEs): European Society of Gastrointestinal Endoscopy Position Statement - Update 2014. Endoscopy 2015; 47: 80-86

[5] Tanaka S, Kashida H, Saito Y et al. JGES guidelines for colorectal endoscopic submucosal dissection/endoscopic mucosal resection. Dig Endosc 2015; 27: 417-434

[6] Bosman FT, Carneiro F, Hruban RH et al. WHO Classification of Tumours of the Digestive System. World Health Organization Classification of Tumours. Tumors of the stomach Lyon, France.: IARC; 2010: $45-80$

[7] Cotton PB, Eisen GM, Aabakken L et al. A lexicon for endoscopic adverse events: report of an ASGE workshop. Gastrointest Endosc 2010; 71: 446-454

[8] Probst A, Golger D, Anthuber M et al. Endoscopic submucosal dissection in large sessile lesions of the rectosigmoid: learning curve in a European center. Endoscopy 2012; 44: 660-667

[9] Ebigbo A, Probst A et al. Step-Up Training for colorectal and gastric ESD and the challenge of ESD training in the proximal colon: Results from a German Center. Endosc Int Open 2018; 6: E524-E530

[10] Fuccio L, Hassan C, Ponchon T et al. Clinical outcomes after endoscopic submucosal dissection for colorectal neoplasia: a systematic review and meta-analysis. Gastrointest Endosc 2017; 86: 74-86

[11] Gupta S, Miskovic D, Bhandari P et al. A novel method for determining the difficulty of colonoscopic polypectomy. Frontline Gastroenterol 2013; 4: 244-248 